\title{
Comparação entre a pressão inspiratória máxima aferida pelo manovacuômetro digital e pelo dispositivo eletrônico de treinamento muscular inspiratório
}

\author{
Comparison between maximum inspiratory pressure measured by a digital manometer \\ and by an electronic inspiratory muscle training device
}

\author{
Áurea Gonçalves Ferreira ${ }^{1}$, Fabieli Vicente ${ }^{1}$, Ligia dos Santos Roceto Ratti ${ }^{1,2}$, Rodrigo Marques Tonella ${ }^{1,2}$, \\ Antonio Luis Eiras Falcão ${ }^{1}$, Ana Paula Ragonete dos Anjos ${ }^{2} \bowtie$, Luciana Castilho de Figueirêdo ${ }^{1}$ \\ ${ }^{1}$ Curso de Especialização em Fisioterapia Respiratória em UTI Adulto do Hospital de Clínicas da Unicamp. Campinas, SP. \\ 2 Faculdade de Ciências Médicas da Universidade Estadual de Campinas (UNICAMP). Campinas, SP.
}

\section{RESUMO}

Objetivos: Comparar valores de pressão inspiratória máxima (PImáx) aferidos por um manovacuômetro digital e por um dispositivo eletrônico de treinamento muscular inspiratório e avaliar as repercussões hemodinâmicas após as medidas.

Métodos: A amostra foi composta por indivíduos acima de 18 anos, de ambos os sexos, internados na unidade de terapia intensiva, hemodinamicamente estáveis, sem uso de drogas vasoativas ou de sedação, intubados ou traqueostomizados, em processo de desmame da ventilação mecânica. Foram feitas três medidas de PImáx pelos dois equipamentos, com tempo de oclusão de 20 segundos e tempo entre as medidas de cinco minutos. Foram analisados frequência respiratória, pressão arterial média e frequência respiratória antes e após cada medida para cada equipamento utilizado. A análise estatística usou os programas Statistical Analysis System e R Project for Statistical Computing V. 3.1.2, aplicando os testes ANOVA e Wilcoxon.

Resultados: Foram incluídos no estudo 58 pacientes. A média das PImáx obtidas com o manovacuômetro digital foi -46,22 centímetros de água $\left(\mathrm{cmH}_{2} \mathrm{O}\right)$, enquanto a média obtida com o dispositivo de TMI foi - $13,15 \mathrm{cmH}_{2} \mathrm{O}(\mathrm{p}<0,001)$. A frequência cardíaca apresentou aumento na comparação antes e após todas as medidas em ambos os dispositivos $(\mathrm{p}<0,0001)$. A pressão arterial média apresentou diferença estatisticamente significativa somente entre antes e após a primeira medida obtida pelo manovacuômetro digital, e entre antes e após a segunda medida obtida com o dispositivo de treinamento muscular inspiratório $(p<0,001)$. A frequência respiratória apresentou variação significativa entre antes e após as três medidas em ambos dispositivos $(\mathrm{p}<0,0001)$. Os valores das variáveis hemodinâmicas após as medidas de PImáx permaneceram dentro dos limites da normalidade.

Conclusões: O manovacuômetro digital registrou uma PImáx superior à registrada pelo dispositivo eletrônico de treinamento muscular inspiratório. Ambos os dispositivos alteraram os valores das variáveis hemodinâmicas, que entretanto permaneceram dentro da normalidade e sem repercussão clínica.

DESCRITORES: doenças respiratórias; insuficiência respiratória; unidade de terapia intensiva; desmame do respirador.

\section{ABSTRACT}

Aims: To compare maximum inspiratory pressure (MIP) measured by a digital manometer and by an inspiratory muscle training (IMT) device and to evaluate hemodynamic changes after measurements.

Methods: The sample included male and female individuals older than 18 years admitted to an intensive care unit who were hemodynamically stable, not being treated with vasoactive drugs or sedated, intubated or tracheostomized, and who were in the process of being weaned from mechanical ventilation. MIP was measured by both devices on three different occasions, with an occlusion time of 20 seconds and a 5 -minute interval between measurements. The following parameters were assessed: respiratory rate, mean arterial pressure, and respiratory rate before and after each measurement by each device. The statistical analysis was made in the Statistical Analysis System and the R Project for Statistical Computing V. 3.1.2 softwares, using the ANOVA and the Wilcoxon tests.

Results: Fifty-eight patients were included in the study. The mean value obtained for MIP was -46.22 centimeters of water $\left(\mathrm{cmH}_{2} \mathrm{O}\right)$ in the digital manometer and $-13.15 \mathrm{cmH}_{2} \mathrm{O}(\mathrm{p}<0.001)$ in the IMT device. Heart rate showed a significant increase $(\mathrm{p}<0.0001)$ both before and after all measurements in both devices. Mean arterial pressure showed a statistically significant difference only before and after the first measurement by the digital manometer and before and after the second measurement by the IMT device $(\mathrm{p}<0.001)$. The respiratory rate oscillated significantly before and after the three measurements by both devices $(\mathrm{p}<0.0001)$. The hemodynamic parameters remained within reference values after MIP measurements.

Conclusions: The digital manometer recorded a higher MIP than that measured by the IMT device. The hemodynamic parameter values oscillated in both devices, but they remained within the normal range and were not clinically significant.

KEY WORDS: respiratory tract diseases; respiratory failure; intensive care unit; ventilator weaning.

Recebido: dezembro, 2015

Aceito: março, 2016

Este artigo está licenciado sob forma de uma licença Creative Common Atribuição 4.0 Internacional, que permite uso irrestrito, distribuição e reprodução em quaquer meio, desde que a publicação original seja corretamente citada. 
Abreviaturas: PImáx, pressão inspiratória máxima; TMI, dispositivo de treinamento muscular inspiratório; FR, frequência respiratória; FC, frequência cardíaca; PAM, pressão arterial média; DP, desvio padrão; rpm, movimentos respiratórios por minuto; $\mathrm{mmHg}$, milímetros de mercúrio; $\mathrm{cmH}_{2} \mathrm{O}$, centímetros de água.

\section{INTRODUÇÃO}

O desmame do respirador pode ser definido como o processo de transição da ventilação mecânica artificial para a ventilação espontânea. Essa fase é considerada crítica na assistência ventilatória em terapia intensiva [1,2]. A ventilação mecânica por um tempo prolongado poderá desencadear alterações na função da musculatura respiratória, principalmente no diafragma, músculo que constantemente é recrutado na presença de algum distúrbio respiratório, o que pode desencadear a fadiga muscular. Para avaliar a capacidade da musculatura respiratória em manter a ventilação espontânea, vários índices preditivos são recomendados na literatura, levando em consideração o equilíbrio entre a carga imposta à musculatura respiratória e a capacidade de sustentar essa carga, indicando o sucesso ou não no desmame [3-5].

Dentre as medidas preditivas, a medida da pressão inspiratória máxima (PImáx) pode ser realizada com o objetivo de avaliar a força muscular inspiratória do paciente. Black e Hyatt [6], em 1969, descreveram uma forma prática de obter a PImáx por meio de um manovacuômetro analógico [6-8]. Em 1986, Marini et al. [9] associaram o manovacuômetro analógico a uma válvula unidirecional inspiratória com o objetivo de induzir inspirações forçadas máximas, atingindo assim um volume próximo do residual. Esses autores descreveram o método como forma de obtenção da PImáx em pacientes sob ventilação mecânica [9]. Estudos mais recentes de Souza et al. [10] sugerem que para uma medida confiável da PImáx em pacientes orientados e colaborativos, seria necessário um tempo de oclusão da válvula unidirecional em torno de 40 a 60 segundos [8-10].

Com os avanços da medicina associados à tecnologia, foram desenvolvidos equipamentos que podem mensurar a PImáx de forma precisa e informatizada. No Brasil, o Núcleo de Estudos e Pesquisas em Engenharia Biomédica (NEPEB) da Escola de Engenharia da Universidade Federal de Minas Gerais desenvolveu um manovacuômetro digital com escala de até 300 centímetros de água $\left(\mathrm{cmH}_{2} \mathrm{O}\right)$ para medida da força muscular inspiratória e capacidade de armazenamento eletrônico de dados [7]. Kulkarni et al. [8] referem que existem dispositivos eletrônicos de treinamento muscular inspiratório e expiratório capazes de acompanhar o treinamento e também monitorar o ganho de força muscular inspiratória, inclusive com possibilidade de armazenamento dos dados em computador [8].

O presente estudo teve como objetivo comparar valores de PImáx obtidos por meio do manovacuômetro digital e de um dispositivo de treinamento muscular inspiratório (TMI) e avaliar as variações hemodinâmicas e respiratórias antes e após as medidas.

\section{MÉTODOS}

Foi realizado um estudo prospectivo em pacientes internados na unidade de terapia intensiva (UTI) do Hospital das Clinicas da Universidade Estadual de Campinas (HC/UNICAMP). O estudo foi aprovado pelo Comitê de Ética e Pesquisa da instituição, sob o parecer no 403/626, e um Termo de Consentimento Livre e Esclarecido foi explicado e assinado pelo familiar responsável por cada paciente incluído no estudo.

Foram selecionados 58 pacientes de ambos os sexos, com idade superior a 18 anos, estáveis hemodinamicamente, sem uso de drogas vasoativas ou com dose mínima (dopamina, dobutamina ou noradrenalina $\leq$ que $5 \mathrm{mcg} / \mathrm{kg} / \mathrm{min}$ ), sem uso de sedativos (exceto analgésicos) e temperatura corpórea entre $36,5^{\circ} \mathrm{C}$ e $38,5^{\circ} \mathrm{C}$. Todos os pacientes utilizavam via aérea artificial, com tudo orotraqueal ou traqueostomia, e estavam em processo de desmame da ventilação mecânica em modo ventilatório espontâneo com parâmetros ajustados de pressão de suporte $\leq 20 \mathrm{cmH}_{2} \mathrm{O}$, pressão positiva expiratória final $\leq 8 \mathrm{cmH}_{2} \mathrm{O}$ e fração inspirada de oxigênio $\leq 0,4$; ou em nebulização por período intermitente intercalado com o modo espontâneo referido acima. Em ambos os processos de desmame, os pacientes incluídos apresentavam saturação periférica de oxigênio $\geq 95 \%$. Foram excluídos do estudo pacientes que apresentavam contraindicações para a realização de manobra inspiratória forçada, tais como hipertensão intracraniana e instabilidade da caixa torácica, e aqueles que apresentaram sinais clínicos de fadiga muscular inspiratória ou qualquer sintoma de intolerância na avaliação inicial: frequência respiratória (FR) >35 movimentos por minuto (mpm), sinais de desconforto respiratório, uso dos músculos acessórios, respiração com movimento toracoabdominal paradoxal, saturação periférica de oxigênio $<90 \%$ e/ou frequência cardíaca (FC) $>120$ batimentos por minuto (bpm). 
Para a definição do equipamento que seria escolhido primeiro, o manovacuômetro digital MVD300 (Globalmed, Rio Grande Sul, Brasil) ou o dispositivo TMI (POWERbreathe NCS, São Paulo, Brasil), foi realizado um sorteio com envelopes fechados, antecedendo cada coleta.

Previamente às medidas, foram realizadas manobras de higiene brônquica, com aspiração traqueal, posicionamento no leito com cabeceira em $45^{\circ}$ (posição de Fowler) e ventilação com uma fração inspirada de oxigênio de 1,0 durante um minuto. Foram monitorizados e anotados antes e após o procedimento a FC, a FR e a pressão arterial média (PAM), através de um monitor (Philips Intelli Vue MP $40^{\circledR}$ ).

Em seguida, foi aplicado o método de aferição da PImáx conforme descrito por Marini et al. [9] em 1986 e Monteiro et al. [3] em 2004, pela oclusão da via inspiratória por tempo variável. Para a obtenção da PImáx foi acoplada uma válvula unidirecional em cada equipamento, com tempo de oclusão de 20 segundos $[3,9]$. O procedimento foi realizado três vezes, sem uso de estímulo verbal, por um mesmo avaliador e com intervalo de um minuto entre as medidas. Foi mantido um intervalo de 10 minutos para obtenção da PImáx entre os equipamentos. Para os cálculos foi considerado o valor médio dessas três medidas.

A análise descritiva foi realizada por meio de medidas de posição e dispersão para as variáveis contínuas e tabelas de frequências para variáveis categóricas. Para análise estatística foram utilizados os programas SAS (Statistical Analysis System) e R (The R Project for Statistical Computing V. 3.1.2). Para comparação de medidas contínuas avaliadas em três momentos na mesma unidade amostral, foi aplicado o teste de Wilcoxon. Para comparação entre os dois grupos para medidas repetidas foram aplicados o teste ANOVA (análise de variância). O nível de significância adotado foi de $5 \%$.

\section{RESULTADOS}

Foram incluídos no estudo 58 pacientes, sendo $37(63,8 \%)$ do sexo masculino e $21(36,2 \%)$ do sexo feminino, com média de idade de 50,14 $\pm 15,36$ anos, tempo de internação de $16 \pm 14,59$ dias e tempo médio de ventilação mecânica de $15,05 \pm 14,63$ dias. A Tabela 1 demonstra as características gerais da amostra estudada.

A Figura 1 demonstra os valores das médias da PImáx obtidas em ambos os dispositivos. A média dos valores da PImáx obtidas com o manovacuômetro digital e com o dispositivo para TMI não apresentaram variações significativas entre as três medidas, em cada equipamento $(p=0,263)$. O valor da PImáx nas três aferições consecutivas, parece ter sido homogêneo. Entretanto, em análise comparativa entre os valores obtidos em relação ao dispositivo utilizado, houve diferença. A média das pressões obtidas com o manovacuômetro digital foi de -46,22 $\mathrm{cmH}_{2} \mathrm{O}$, enquanto a média obtida com dispositivo de TMI foi $-13,15 \mathrm{cmH}_{2} \mathrm{O}(\mathrm{p}<0,001)$.

Tabela 1. Características gerais dos 58 pacientes participantes do estudo, 37 homens e 21 mulheres internados em unidade de terapia intensiva, em processo de desmame de ventilação mecânica.

\begin{tabular}{|lcc}
\hline \multicolumn{1}{c}{ Variáveis } & n & $\begin{array}{c}\text { Média } \pm \\
\text { desvio padrão }\end{array}$ \\
\hline Idade (anos) & 58 & $50,14 \pm 15,36$ \\
\hline Tempo de internação hospitalar (dias) & 58 & $16 \pm 14,59$ \\
\hline Tempo de utilização de ventilação mecânica (dias) & 58 & $15,05 \pm 14,63$ \\
\hline Tempo de intubação orotraqueal (dias) & 57 & $7,97 \pm 0,24$ \\
\hline Tempo de traqueostomia (dias) & 22 & $8,02 \pm 0,24$ \\
\hline Escore APACHE II (pontuação) & 58 & $17,43 \pm 7,99$ \\
\hline Mortalidade (\%) & 58 & $29,21 \pm 20,04$ \\
\hline Mortalidade ajustada por escore de gravidade (\%) & 56 & $17,63 \pm 18,78$ \\
\hline
\end{tabular}

APACHE II, sistema de pontuação de mortalidade em unidade de terapia intensiva (Knaus WA et al., Crit Care Med. 1985Oct;13:818-29).

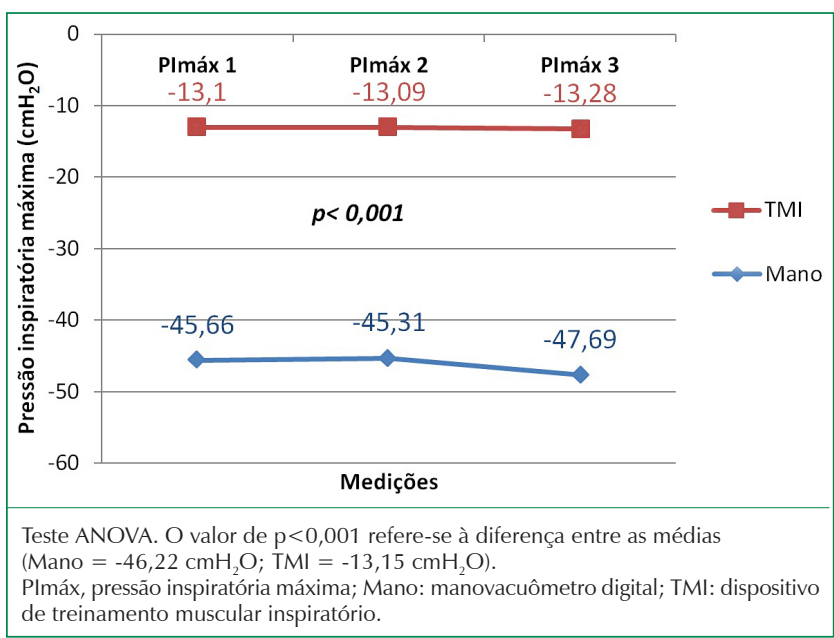

Figura 1. Valores da pressão inspiratória máxima obtidos nas três medidas em cada dispositivo (manovacuômetro digital e dispositivo eletrônico de treinamento muscular inspiratório), em 58 pacientes adultos em processo de desmame de ventilação mecânica.

A Figura 2 demonstra os valores obtidos da FC nos momentos antes e após as três medidas realizadas com o manovacuômetro digital e as três medidas realizadas com o dispositivo para TMI. Houve variações dos valores de FC em todos os procedimentos 
realizados com ambos os dispositivos. Embora a diferença estatística dos valores entre os dispositivos nos momentos antes e após o procedimento, fosse estatisticamente significativa, as variações foram dentro da normalidade.

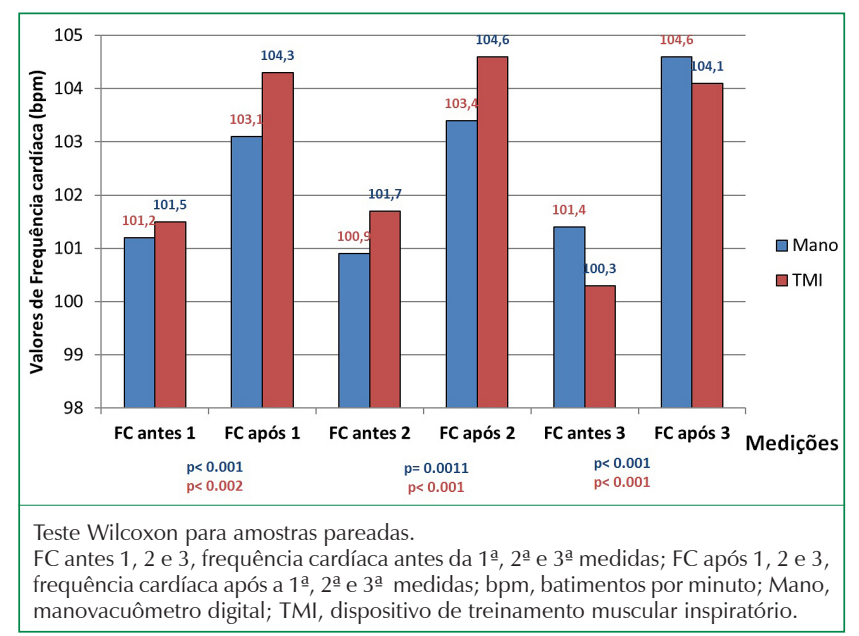

Figura 2. Comparação dos valores da frequência cardíaca obtidos antes e após as avaliações feitas com um manovacuômetro digital e um dispositivo eletrônico de treinamento muscular inspiratório, em 58 pacientes em processo de desmame de ventilação mecânica.

A PAM apresentou diferença significativa entre antes e após a primeira medida de PImáx obtida pelo manovacuômetro digital (respectivamente $86,8 \pm$ $12,9 \mathrm{mmHg}$ e $91,6 \pm 14,3 \mathrm{mmHg}, \mathrm{p}<0,001)$. Quando usado o dispositivo para TMI, a PAM foi estatisticamente diferente entre antes e após a segunda medida (respectivamente $89,4 \pm 12,2$ e $91,1 \pm 12,5, \mathrm{p}<0,001)$ (Figura 3).

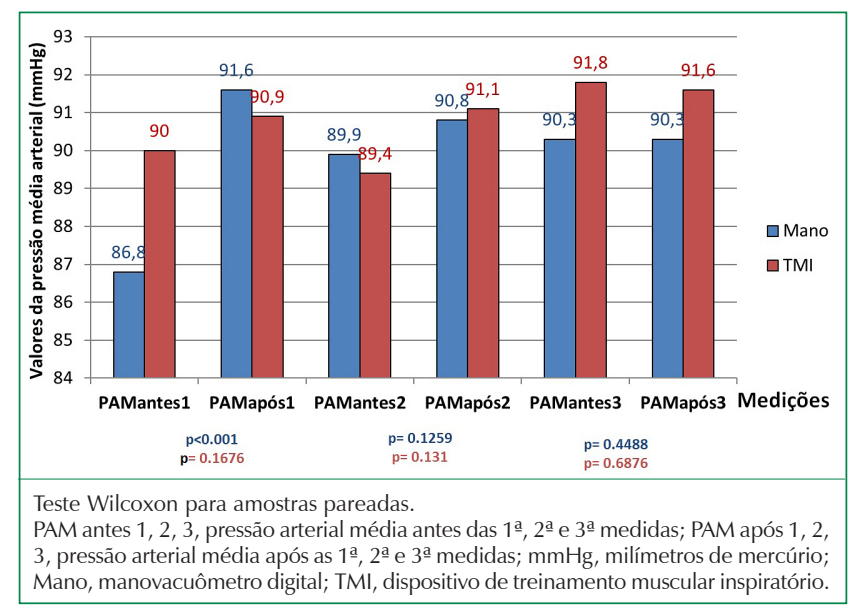

Figura 3. Comparação dos valores da pressão arterial média obtidos antes e após as avaliações feitas com um manovacuômetro digital e um dispositivo eletrônico de treinamento muscular inspiratório, em 58 pacientes em processo de desmame de ventilação mecânica.
Os valores da FR apresentaram diferença estatística significativa entre os momentos antes e após em todas as medidas realizadas para ambos dispositivos, com aumento após a aferição da PImáx, porém dentro dos valores de normalidade (Figura 4).

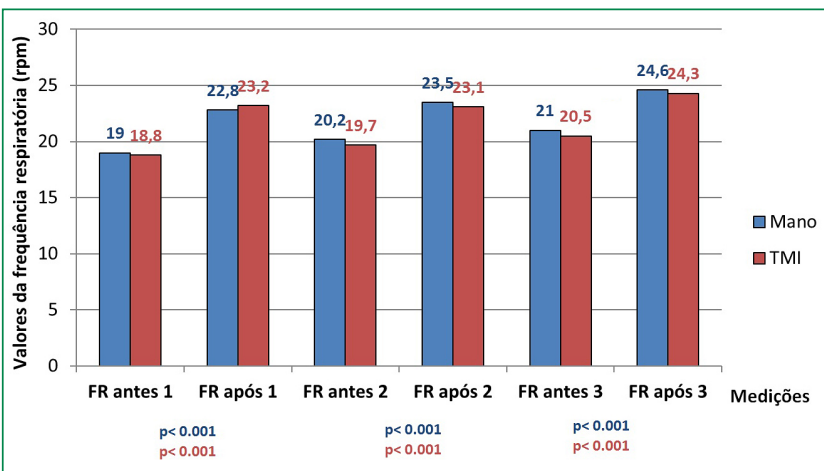

Teste de Wilcoxon para amostras pareadas.

FR antes 1, 2, 3, frequência respiratória antes das $1 \underline{a}$, $2^{\underline{a}}$ e $3^{\underline{a}}$ medidas; FR após $1,2,3$, frequência respiratória após as $1 \underline{a}, 2^{\underline{a}}$ e $3^{\underline{a}}$ medidas; rpm, respirações por minuto; Mano, manovacuômetro digital; TMI, dispositivo de treinamento muscular inspiratório e expiratório.

Figura 4. Comparação dos valores da frequência respiratória obtidos antes e após as avaliações feitas com um manovacuômetro digital e um dispositivo eletrônico de treinamento muscular inspiratório, em 58 pacientes em processo de desmame de ventilação mecânica.

\section{DISCUSSÃO}

Uma das principais metas de ordem prática por ocasião da elaboração do presente estudo foi comparar diferentes dispositivos de aferição da PImáx em pacientes intubados ou traqueostomizados e avaliar alterações hemodinâmicas, já que não existe um consenso na literatura, ou recomendações sobre o melhor equipamento para aferição da PImáx de maneira rápida, eficaz, prática e confiável.

A comparação dos dispositivos para aferição da PImáx em pacientes intubados ou traqueostomizados pode trazer como benefícios, menor tempo de utilização da ventilação mecânica e de hospitalização e diminuir o índice de mortalidade, devido à não subestimação da PImáx. Desta forma, os pacientes em ventilação mecânica, especialmente aqueles que estão em processo de desmame, são os mais beneficiados.

A avaliação da PImáx tem como objetivos predizer o sucesso no desmame da ventilação mecânica, avaliar a força muscular respiratória e calcular a carga de treinamento muscular inspiratória e expiratória. A maioria dos estudos relacionados demonstram $80 \%$ de sensibilidade, porém com $25 \%$ de especificidade, ou seja, é possível que pacientes que apresentam falha no desmame não tenham necessariamente uma 
PImáx reduzida. Além disso, a reprodutibilidade é questionável, devido à diversidade de métodos e a dificuldade para a padronização da técnica; e, ainda, os estudos não contemplam populações homogêneas [11,12].

Os resultados encontrados no presente estudo demonstram que as medidas de PImáx realizadas com o manovacuômetro digital e o dispositivo de TMI com o método convencional de válvula unidirecional ocluída por 20 segundos, apresentaram diferenças significativas, ou seja, os valores da PImáx obtidos com o dispositivo de TMI foram menores do que os obtidos com o manovacuômetro digital. Corroborando com o presente estudo, Lima et al. [13] notaram que os valores obtidos, tanto da PImáx quanto da pressão expiratória máxima com o manovacuômetro digital, foram predominantemente superiores aos valores obtidos com o manovacuômetro analógico. Em contrapartida, Saad et al. [14] concluíram que não houve diferença estatística significativa entre os valores obtidos com o manovacuômetro digital e o analógico.

Apesar da medida da PImáx ainda não possuir correlação clara com o sucesso no desmame da ventilação mecânica ou o desfecho clínico dos pacientes críticos, acredita-se que maiores valores de PImáx possam estar associados com uma melhor ventilação pulmonar, depuração das vias aéreas e melhores resultados em indivíduos em processo de desmame da ventilação mecânica [3].

A proposta do presente estudo também foi avaliar as repercussões da manobra prolongada nas variáveis hemodinâmicas dos pacientes. Quando comparadas as variáveis nos momentos antes e após as três medidas realizadas em cada dispositivo, foram encontradas diferenças significativas da PImáx em relação às variáveis $\mathrm{FC}$ e FR nas três medidas de ambos os dispositivos. Em síntese, independente do dispositivo, a FC e a FR dos pacientes aumentaram, quando comparadas antes e o após as medidas da PImáx. A PAM apresentou variações com diferença significativa, porém dentro dos valores de normalidade e sem repercussão clínica. O impacto evidenciado nas variáveis $\mathrm{FC}$ e FR não se traduziu necessariamente em alterações clínicas, em consonância com dados da literatura, que consideram como significância clínica o aumento ou diminuição de $20 \%$ nos valores de PAM ou aumento de $10 \%$ da FC em repouso [15-18]. Acredita-se que a mensuração da PImáx em relação às variáveis FC e FR foi segura em ambos os dispositivos utilizados. Filho et al. [4] também não observaram diferença significativa após mensuração da PImáx em relação às variáveis FC, FR e PAM.

Não foi objetivo deste estudo realizar um levantamento sobre sucesso e falha no desmame ventilatório dos pacientes. O estudo não tem poder suficiente para detectar superioridade entre os tempos de oclusão, visto que não foi realizado acompanhamento do desfecho no desmame da ventilação mecânica dos pacientes envolvidos no estudo. Estudos com amostragem maior, diferentes metodologias, com outros tipos de comparações de dispositivos e diferentes métodos de mensuração da pressão inspiratória máxima devem ser realizados.

Nas condições testadas, os valores das pressões inspiratórias máximas obtidas por um manovacuômetro digital e por um dispositivo de TMI foram diferentes entre si, sendo os valores superiores no manovacuômetro digital, sugerindo mais confiança e veracidade desse dispositivo para aferir a PImáx em pacientes intubados ou traqueostomizados. Em relação às variações hemodinâmicas, ambos os dispositivos alteraram os valores das variáveis, porém, estas permaneceram dentro dos valores de normalidade e sem repercussão clínica.

\section{NOTA}

Declaração de conflitos de interesse

Os autores declaram não haver conflitos de iteresse relevantes ao conteúdo deste estudo.

\section{REFERÊNCIAS}

1. Parreira VF, França DC, Zampa CC, Fonseca MM, Tomich GM, Britto RR. Maximal respiratory pressures: actual and predicted valuesin healthy subjects. Rev Bras Fisioter. 2007;11(5):361-8. http://dx.doi.org/10.1590/S1413-35552007000500006

2. Passarelli RCV, Tonella RM, Souza HCD, Gastaldi AC. Evaluation of Inspiratory Muscular Force (Plmáx) during Weaning from Mechanical Ventilation in Neurological Patients in the Intensive Care Unit. Fisioter Pesq. 2011;18(1):48-53. http://dx.doi.org/10.1590/ S1809-29502011000100009

3. Monteiro LS, Veloso CA, Araújo S, Figueiredo LC, Terzi RGG. Comparação de dois métodos de mensuração da pressão inspiratória máxima com o uso de uma válvula unidirecional. Rev Bras de Ter Intensiva. 2004;16:74-7. 
4. Filho GRP, Reis HFC, Almeida MJ, Andrade WS, Rocha RLS, Leite PA. Comparison and effects of two different airway occlusion times during measurement of maximal inspiratory pressure in adult intensive care unit neurological patients. Rev Bras Ter Intensiva. 2010;22(1):33-9. http://dx.doi.org/10.1590/S0103-507X2010000100007

5. Souza C, Da Silva CT Jr, Lugon JR. Evaluation of the inspiratory pressure using a digital vacuometer in mechanically ventilated patients: analysis of the time to achieve the inspiratory peak. Respir Care. 2012 Feb;57(2):257-62. http://dx.doi.org/10.4187/respcare.01228

6. Black LF, Hyatt RE. Maximal Respiratory pressures: Normal Valus and Relationship To Age and Sex. Am Rev Respir Dis. 1969 May;99(5):696-702.

7. Ferreira JL, Nadja C, Oliveira Júnior M, Vasconcelos FH, Parreira V, Tierra-Criollo CJ. Medidor de pressões respiratórias máximas: calibração e cálculo da incerteza. SBA Controle \& Automação. 2010;21(6):589-97.

8. Kulkarni SR, Fletcher E, McConnell AK, Poskitt KR, Whyman MR. Pre-operative inspiratory muscle training preserves postoperative inspiratory muscle strength following major abdominal surgery - a randomised pilot study. Ann R Coll Surg Engl. 2010 Nov;92(8):700-7. http://dx.doi.org/10.1308/003588410X12771863936648

9. Marini JJ, Smith TC, Lamb V. Estimation of inspiratory muscle strength in mechanically ventilated patients: the measurement of maximal inspiratory pressure. J Crit Care. 1986 Mar;1(1):32-8. http://dx.doi.org/10.1016/S0883-9441(86)80114-9

10. Souza LC. Impact of using a digital vacuum gauge and check valve on respiratory indices predictors of weaning. Nitéroi: Universidade Federal Fluminense; 2011.

11. Silva BAK, Souza JKD, Pereira DM, Aydos RD, Carvalho PTC, Reis FA. Correlação entre pressão inspiratória máxima, ventilação pulmonar e tempo de ventilação em pacientes ventilados no modo de pressão de suporte. ConScientia e Saúde. 2008;7(3):379-84. http:// dx.doi.org/10.5585/conssaude.v7i3.1370

12. Caruso P, Albuquerque LP, Santana PV, Cardenas LZ, Ferreira JG, Prina E. Métodos diagnósticos para avaliação da força muscular inspiratória e expiratória. J Bras Pneumol, 2015;41(2):110-23. http://dx.doi.org/10.1590/S1806-37132015000004474

13. Lima, L. P. Mensuração das pressões respiratórias máximas com equipamentos analógico e digital. In: $5^{\circ}$ Amostra Acadêmica UNIMEP. Piracicaba; 2007

14. Saad IAB, Santos BP, Paganini C, Guidetti EL, Silva YB, Toro IFC, Zambon L. Comparison between maximal respiratory pressures obtained from digital and analog manovacuometers in 120 Brasilian patients with lung disease. Eur Respir J. 2014;44(Suppl58):4284.

15. Clanton TL, Diaz PT. Clinical assessment of the respiratory muscles. Phys Ther. 1995 Nov;75(11):983-95.

16. Stiller K, Phillips A. Safety aspects of mobilising acutely ill in patients. Physiother Theory Pract. 2003;19(4):239-57. http://dx.doi org/10.1080/09593980390246751

17. Stiller K, Philips AC, Lambert P. The safety of mobilisation and its effect on haemodynamic and respiratory status of intensive care patients. Physiother Theory Pract. 2004;20(3):175-85. http://dx.doi.org/10.1080/09593980490487474

18. Stiller K. Safety issues that should be considered when mobilizing critically ill patients. Crit Care Clin. 2007 Jan;23(1):35-53. http:/ dx.doi.org/10.1016/j.ccc.2006.11.005

19. Knaus WA, Draper EA, Wagner DP, Zimmerman JE. APACHE II: a severity of disease classification system. Crit Care Med. 1985 Oct;13(10):818-29. http://dx.doi.org/10.1097/00003246-198510000-00009 